\title{
COMMISSIONING THE UPGRADED RF BUNCHER AT THE LANSCE PROTON STORAGE RING*
}

\author{
J. Lyles, A. Browman, R. Kustom ${ }^{\dagger}$, Los Alamos National Laboratory \\ Los Alamos, New Mexico 87545 USA
}

\section{Abstract}

The 2.8 Megahertz (MHz) cathode-follower RF amplifier system and ferrite-loaded gap assembly for the Proton Storage Ring (PSR) were improved as part of the Los Alamos Neutron Science Center (LANSCE) upgrade for a Short Pulse Spallation Source (SPSS) [1]. The commissioning of PSR commenced in July of 1998. The performance of the cathode-follower amplifier system and gap was measured with as much as five microcoulombs $(\mu \mathrm{C})$ of circulating beam, approximately forty Amperes of peak current before extraction. The output impedance of the cathode follower was estimated to be approximately eleven Ohms. This was calculated from beam-induced voltage on the gap with the amplifier operating class A (full conduction of plate current), with RF drive off. Measurements showed the perturbation of the gap voltage with beam loading to be minimal. The improved RF system handled the beam loading well. A new phase feedback control system corrected for three degrees of phase shift from unloaded to fully loaded beam conditions, with $18 \mathrm{kV}$ of peak RF voltage across the gap. The amplitude feedback controller made 300 volts of correction for droop, also minimal because of the low output impedance at the gap. Beam instability at PSR with higher current was controlled, as expected, with higher voltage [2]. Analysis of these and other measurements are presented.

\section{RF BUNCHER REQUIREMENTS}

The PSR is a fast-cycling high current ring designed to accumulate beam over a macropulse from the LANSCE linac, with multi-turn injection. Stacking these protons over approximately 2100 turns allows very high charge densities to be stored. A single-turn extracted pulse (250 ns) of high current drives the new SPSS target, to produce neutrons of the desired characteristics. In January of 1999 LANSCE achieved $3.1 \times 10^{13}$ at $20 \mathrm{~Hz}$, with an average current of 100 microamperes $(\mu \mathrm{A})$, a 43 percent increase over 1998 operation. The SPSS end goal is to double this current, to $200 \mu \mathrm{A}$.

The PSR uses a RF buncher system to control longitudinal beam distribution. The improved RF buncher is described in a companion paper [1] in these proceedings.

\subsection{Increasing Bunching Factor}

This project raised the buncher gap voltage to $18 \mathrm{kV}$ peak at $2.8 \mathrm{MHz}$ (exactly 2.79513 ), and designed the amplifiers to also be usable at $\mathrm{h}=2$, or $5.6 \mathrm{MHz}$. Continuing to raise the $2.8 \mathrm{MHz}$ bunching voltage alone would eventually result in excess space charge tune shift. It would be better for the peak charge density to be accumulated in a long flat bunch to allow higher beam current in the ring. The application of a second harmonic voltage of the proper phase can increase bunching factor from 0.35 to 0.5 , creating this flat bunch. Figure 1 shows the PSR current during optimal bunching for a single frequency $h=1$ system.

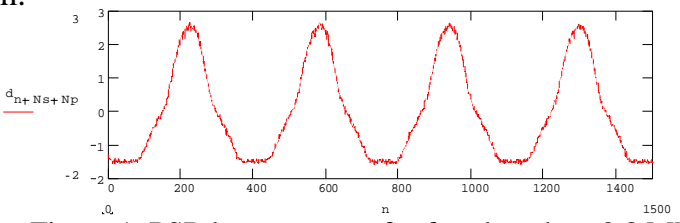

Figure 1. PSR beam current for four bunches, $2.8 \mathrm{MHz}$

\subsection{PSR Instability Threshold}

The PSR has operated just below a fast transverse instability, which has been studied and described [3]. It becomes evident when the beam intensity exceeds about 2 x $10^{13} \mathrm{ppp}$. The buncher RF voltage has demonstrated a strong coupling to the instability threshold level. Because of this, it was expected that increasing the RF voltage would give more latitude for future increases in beam current. This will eventually be limited by space charge factors. At $200 \mu \mathrm{A}$, the goal for PSR is $4.2 \times 10^{13} \mathrm{ppp}$ at $30 \mathrm{~Hz}$.

\section{TEST RESULTS OF RF BUNCHER}

\subsection{High Power RF}

The improved buncher delivered $18 \mathrm{kV}$ peak to the gap in July of 1998. We also operated the new intermediate power amplifier (IPA) at $\mathrm{h}=2$, to demonstrate that it was capable of tuning to $5.6 \mathrm{MHz}$, at full output. Initially the IPA was operated into dummy load for $5 \%$ duty factor, purchased from the Ohm-Weve ${ }^{\circledR}$ Company. It simulated the $2 \times 500 \mathrm{Ohm}$ input resistors plus the capacitive reactances of the grids of the final power amplifier (FPA).

We operated with $5 \%$ duty factor with pulses up to 2.2 ms wide, and rep rates to $30 \mathrm{~Hz}$ with $1.7 \mathrm{~ms}$. The IPA output pi-networks were easily tuned under power. A fast

\footnotetext{
* Work supported by the US Department of Energy

${ }^{\dagger}$ Visiting from Argonne National Laboratory
} 
digitizing oscilloscope (TDS 784D) connected to a pair of internal Jennings capacitive voltage dividers allowed for simple adjustment for 180 degrees between the two IPA output feeders. The value of internal amplifier diagnostics was quickly proven.

\subsection{Low Level RF Control}

The amplitude and phase controls were tested without beam. The improved lower threshold of the amplitude feedback control (AGC) is 500 Volts, whereas it had been difficult to set the level below $2 \mathrm{kV}$. Open loop phase skew of the amplifier was measured while the amplitude was ramped at the input. Across the full range of usable output, about 20 degrees of phase change was measured, in the same direction for both tetrodes.

The IPA was coupled to the FPA with the new interconnections, and the same measurements were made (except for $5.6 \mathrm{MHz}$ ) while driving the beam gap. The ferrite bias was set for $2.8 \mathrm{MHz}$ resonance. A pair of calibrated LANL voltage dividers is mounted within the cavity, and these signals normally provide feedback to the low level RF controls. The differential signal is combined. The RF voltage in figures 3 - 6 were measured this way.

\section{TEST RESULTS WITH BEAM IN PSR}

In September of 1998, beam was provided to commission the upgraded buncher. The low level RF now provides error signals for the amplitude channel and the new phase controller. These signals were monitored while beam was injected. All tests were performed at very low duty factor. Figure 2 shows the errors as $2.5 \mu \mathrm{C}$ of charge is stored. The envelope store mode was used, with 20 pulses (several minutes). The amplitude controller has a correction of about $600 \mathrm{~V}$ pk-pk before extraction, and for droop from the power supplies. The phase controller has one degree of error across the ramp.

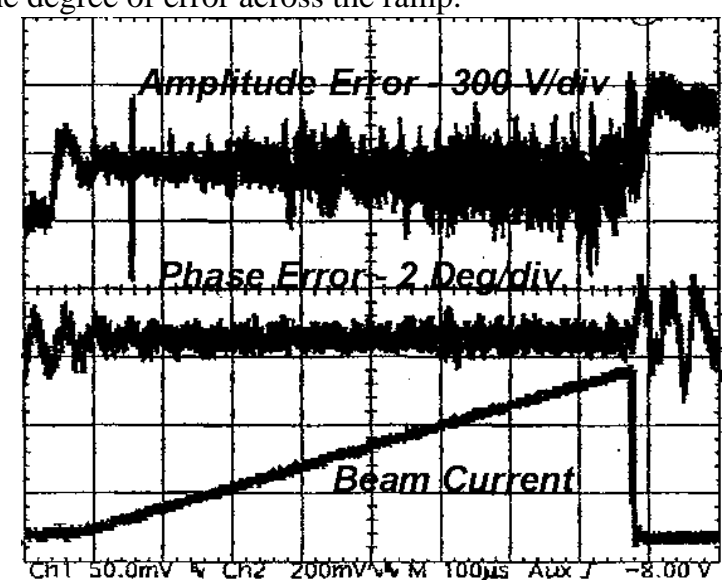

Figure 2. Amplitude and Phase Errors

\subsection{FPA Output Impedance Verification}

This test involved measuring a single cycle of gap voltage near the peak of the ramp, once with beam, and then at the same time period without beam. These were subtracted in the digitizer memory, creating the residual voltage in figure 3 . This is $1.3 \%$ ripple due to the beam current in the gap. This change is too rapid for the AGC to compensate, but it is damped by the low output impedance.

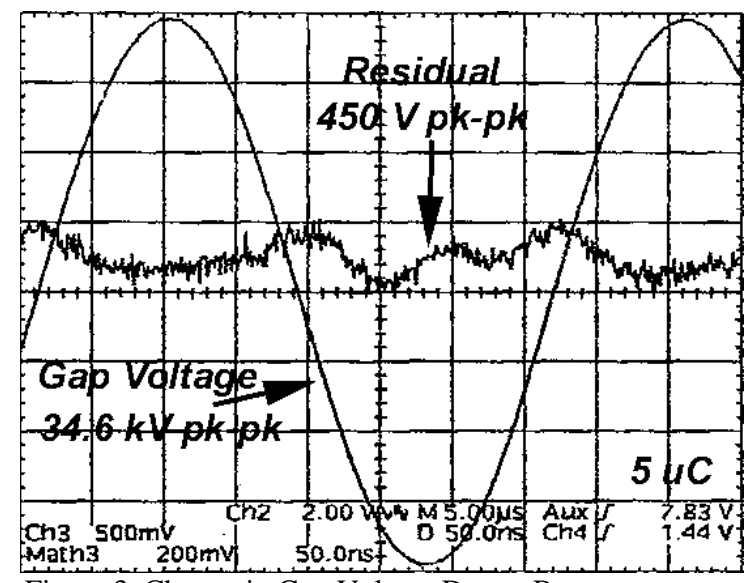

Figure 3. Change in Gap Voltage Due to Beam

Another experiment measured the induced voltage at the gap, when the beam is coasting. The RF drive was off, but the cathode followers were in conduction at 45 Amperes quiescent plate current. The top trace in figure 4 is the induced voltage across the gap, and the bottom trace is the beam current. At extraction, the voltage decays. The voltage was about 11 Volts peak, and the triangular beam current was about 1 Ampere. This gives an estimate of 11 Ohms for $\mathrm{R}_{\mathrm{o}}$ across the gap from the cathode followers.

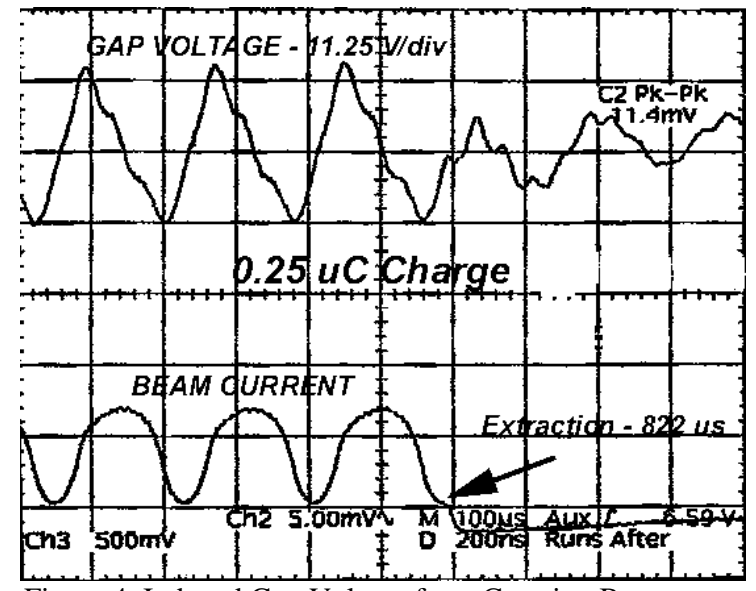

Figure 4. Induced Gap Voltage from Coasting Beam

\subsection{Ferrite Coupling Loop Tests}

In this experiment, the "figure-eight" coupling loop in the cavity is tested with a worst-case scenario. One amplifier is completely shut off, while observing the gap voltage waveform for distortion. This was performed at low buncher voltage, to prevent damage to the termination networks on the ends of loops. This arrangement is described in the companion paper on the buncher improvements. The buncher was set for $3.4 \mathrm{kV}$ peak at 2.8 $\mathrm{MHz}$. Without beam, one cathode follower was put in 
cutoff with the control grid bias supply. There was no visible change in the gap voltage.

The same test was performed with $0.25 \mu \mathrm{C}$ of charge. Figure 5 shows the before and after waveforms, with the beam current superimposed below the voltage trace. Beam was extracted during the interval being digitized. No discernable change in the sinewave was noted, either from beam extraction or from the shutdown of one triode.

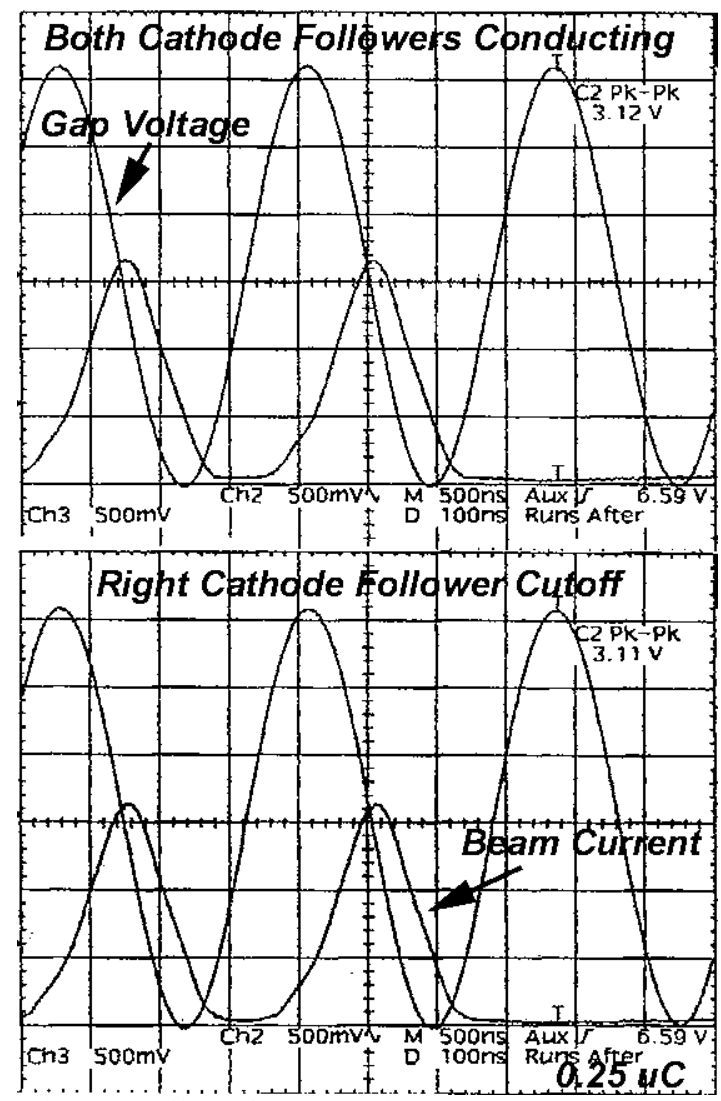

Figure 5. Effect of RF Coupling Loop

It is important to note that this was done with $0.25 \mu \mathrm{C}$ of charge to prevent damage. We would not have been able to do this at $18 \mathrm{kV}$, at full PSR current. At 20 times this amount of charge, it is expected that the loss of one amplifier should be noticeable, as the peak image current is forced through the cathode followers.

\subsection{Tests of Added Second Harmonic Voltage}

This experiment occurred in December of 1998. It required temporary modifications be made to the buncher, in a rapid timeframe. Because of this, the work was done with available components, and the RF duty factor was kept low to prevent damage to the amplifiers and gap. Despite this precaution, several grid resistors were destroyed in the FPA, before we began monitoring the peak dissipation in them. Modeling of the circuit with Spice showed that there would be asymmetry in the cathode currents, and that the phase of $5.6 \mathrm{MHz}$ would have to be carefully monitored to prevent inadvertent peak voltages.
The pi-networks were removed from the IPA, and other modifications were made to eliminate stray resonances in the output, since the high gain tetrodes were now broadbanded. A case in point was the removal of half of the tetrode screen bypass capacitors, when it was discovered that they contributed to a $17 \mathrm{MHz}$ resonance. The ferrite was tuned in-between 2.8 and $5.6 \mathrm{MHz}$, clearly not optimal but manageable with the RF amplifiers.

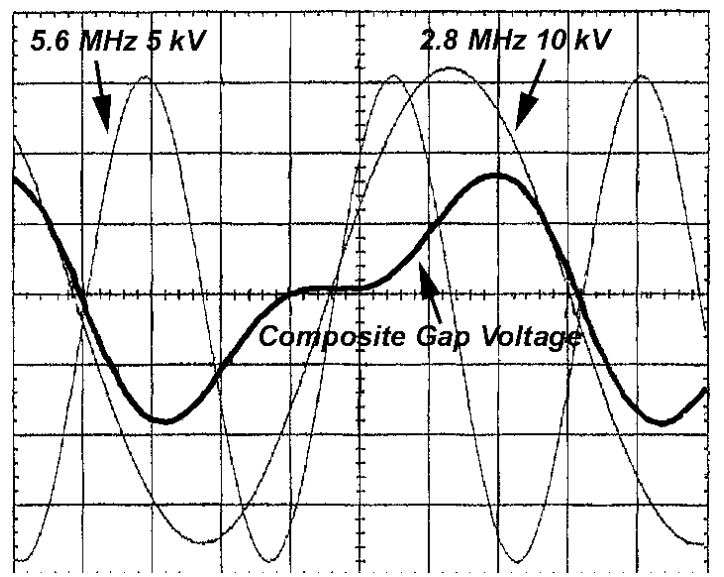

Figure 6. Dual Frequency RF Buncher Voltages

In figure 6, we see the two individual voltages and the composite voltage. This was the highest level of dual frequency operation that was tested. The improved bunching factor was observed in the beam current in PSR, as expected. Figure 7 shows four flat-topped bunches.

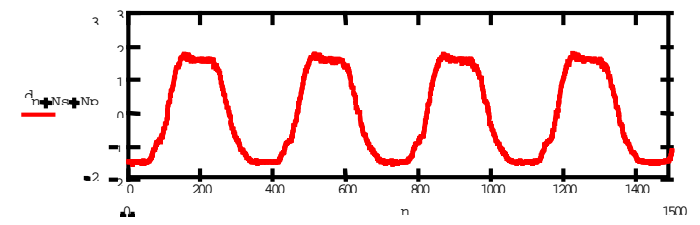

Figure 7. Improved Bunching with Dual Frequency Buncher

\section{CONCLUSION}

The cathode follower RF buncher system at PSR is very robust with high stored beam current. Without the need for high level feedback or turn-to-turn feedforward control it has excellent output regulation. Two harmonics were amplified and the results were favorable for increased charge density. Future plans can be made, based on these experimental results.

\section{REFERENCES}

[1] J. Lyles, J. Davis, "Improvements to the Cathode-Follower RF Amplifier System for the LANSCE Protons Storage Ring Buncher", Proceedings of 1999 Particle Accelerator Conference, New York, NY.

[2] R. Macek, et al. "Overview of Progress on the Improvement Projects for the LANSCE Accelerator and Target Facilities", Proceedings of 1997 Particle Accelerator Conference, Vancouver, BC.

[3] M. Plum, et al. "Recent Experimental Evidence For the Los Alamos Proton Storage Ring Beam Instability", Proceedings of 1997 Particle Accelerator Conference, Vancouver, BC. 